\title{
HIGH SPATIAL RESOLUTION KAO FAR-INFRARED OBSERVATIONS OF THE CENTRAL REGIONS OF INFRARED-BRIGHT GALAXIES
}

\author{
Beverly J. Smith ${ }^{1}$ and P. M. Harvey \\ Department of Astronomy \\ The University of Texas at Austin
}

Austin TX 78712

${ }^{1}$ Now at IPAC/Caltech, MS 100-22, Pasadena CA 91125.

Received:—— Accepted: 


\section{ABSTRACT}

We present new high spatial resolution Kuiper Airborne Observatory (KAO) $50 \mu \mathrm{m}$ and/or $100 \mu \mathrm{m}$ data for 11 infrared-bright galaxies. The sizes of the central far-infrared emitting regions in three of these galaxies, NGC 660, NGC 891, and M 83, agree with those of the central star formation complexes. The $\mathrm{Sb}$ galaxy NGC 7331, which does not have observed star formation in its bulge, has a flat-topped $100 \mu \mathrm{m}$ major axis profile which covers the bulge and inner spiral arms, without a bright central peak or pronounced depression. The remaining seven galaxies, all of which are known to have strong nuclear or circumnuclear star formation, are unresolved or marginally resolved with the KAO, with farinfrared source size limits consistent with the sizes of the central star formation complexes.

Along with these new data, we have tabulated previously published KAO data for 11 other galaxies, and IRAS 60 and $100 \mu \mathrm{m}$ data for the bulges of the large angular size galaxies M 31 and M 81. From the literature, we have compiled optical, near-infrared, and millimeter measurements for the central regions of the entire set of 24 galaxies. We have used this dataset to investigate dust heating and star formation in the central areas of galaxies. We find that $\mathrm{L}(\mathrm{FIR}) / \mathrm{L}(\mathrm{B})$ and $\mathrm{L}(\mathrm{FIR}) / \mathrm{L}(\mathrm{H})$ correlate with $\mathrm{CO}(1-0)$ intensity and $100 \mu \mathrm{m}$ optical depth. Galaxies with optical or near-infrared signatures of OB star formation in their central regions have higher values of $\mathrm{I}(\mathrm{CO})$ and $\tau_{100}$ than more quiescent galaxies, as well as higher far-infrared surface brightnesses and L(FIR)/L(B) and $\mathrm{L}(\mathrm{FIR}) / \mathrm{L}(\mathrm{H})$ ratios. The $\mathrm{L}(\mathrm{FIR}) / \mathrm{L}(\mathrm{H} \alpha)$ ratio does not correlate strongly with $\mathrm{CO}$ and $\tau_{100}$. These data are consistent with a scenario in which OB stars dominate dust heating in the more active galaxies and older stars are important for the more quiescent bulges. Whether or not a galaxy bulge has strong star

formation may be decided by a threshold effect; star forming galaxies have surface 
gas densities above the Kennicutt critical density, while quiescent galaxies have lower values.

\section{Introduction}

It has been known for more than 25 years that galaxies emit a significant amount of their total luminosity at wavelengths longer than $30 \mu \mathrm{m}$, and that this radiation originates from dust in the interstellar medium (Harper and Low 1973; Telesco, Harper, and Loewenstein 1976; Telesco and Harper 1980). The successful IRAS mission in 1983 (IRAS Explanatory Supplement 1988) provided far-infrared luminosities of thousands of galaxies, permitting many investigations into the nature of this far-infrared emission. Two such studies were those of Helou (1986) and Persson and Helou (1987), who suggested a two-component model for the farinfrared emission from galaxies. In this scenario, both OB stars and the general interstellar radiation field contribute to dust heating in galaxies, in proportions differing from galaxy to galaxy. In active galaxies, the AGN may also contribute to dust heating.

Since these publications, there have been many efforts to test and refine this picture. One difficulty has been the relative lack of spatially resolved far-infrared measurements of galaxies. The IRAS resolution is relatively poor $\left(1^{\prime} .5 \times 5^{\prime}\right.$ at 100 $\mu \mathrm{m}$ ), so without special processing, only the most nearby galaxies can be studied in detail (Walterbos and Schwering 1987; Deul 1989; Schwering and Israel 1989; Schwering 1989; Rice et al. 1990; Gallagher et al. 1991). The development of the HiRes deconvolution method for IRAS data (Aumann, Fowler, and Melnyk 1990) has allowed studies of more distant galaxies with an effective resolution of $\sim 80^{\prime \prime}$ at $100 \mu \mathrm{m}$ (Marston 1989; Rand, Kulkarni, and Rice 1992; Devereux and Young 1992, 1993; Rice 1993; Surace et al. 1993; Devereux and Scowen 1994; Marsh and Helou 1995). 
To obtain information at smaller physical scales or in more distant galaxies even higher resolution is needed. To this end, we have conducted a program of far-infrared observations of galaxies using the University of Texas far-infrared photometer systems on the Kuiper Airborne Observatory (KAO). These systems have a $100 \mu \mathrm{m}$ beamsize of approximately $30^{\prime \prime} \times 40^{\prime \prime}$. In a series of previous papers, we have presented KAO far-infrared data for a number of galaxies (Joy et al. 1986, 1987, 1988, 1989; Lester et al. 1986a, 1987, 1995; Brock et al. 1988; Smith et al. 1991, 1994, 1995). In these studies we have identified circumnuclear rings of far-infrared emission (Lester et al. 1986a; Smith et al. 1991), resolved central star formation complexes (Lester et al. 1987; Joy et al. 1987, 1988; Smith et al. 1995), separated bulge and spiral arm emission in normal galaxies (Smith et al. 1991, 1994), resolved the far-infrared emission from a distant giant elliptical galaxy (Lester et al. 1995), separated a close galaxy pair (Joy et al. 1989), and obtained stringent limits on the sizes of the far-infrared emitting regions in luminous galaxies (Joy et al. 1986; Brock et al. 1988). For a more detailed review of these publications, see Lester (1995).

In the current paper, we present new KAO data for 11 additional galaxies. We combine these new data with previously-published KAO data for 11 galaxies, along with IRAS far-infrared data for the bulges of M 31 and M 81. For the entire sample of 24 galaxies, we compare the far-infrared data with published optical, near-infrared, and millimeter observations to investigate dust heating and star formation in the bright central regions of galaxies.

\section{The Observations}

The observed galaxies are given in Table 1, along with their positions, distances, morphological and spectral types, blue magnitudes, and optical diameters. The far-infrared data were obtained with the KAO $0.9 \mathrm{~m}$ telescope during the period 1987 - 1994, flying from either Moffett Field, California, Hickam 
Air Force Base, Hawaii, or Christchurch, New Zealand. Ten of the 11 galaxies were observed at $100 \mu \mathrm{m}$, and three were observed at $50 \mu \mathrm{m}$. Details on the observations of individual galaxies are given in Table 2.

The observations were made with either a $8 \times 1$ bolometer array (Low et al. 1987; Smith et al. 1991) or a $10 \times 2$ element array (Smith et al. 1994). For both these systems, the approximate rms noise level reached in an hour is 0.5 Jy. The $100 \mu \mathrm{m}$ beamsizes of these systems are approximately $25^{\prime \prime} \times 35^{\prime \prime}(8$ channel system) or $31^{\prime \prime} \times 41^{\prime \prime}(20$ channel system), varying slightly from detector to detector, with the short axis of the beam aligned along the long axis of the array. The $50 \mu \mathrm{m}$ beamsize for both systems is approximately $18^{\prime \prime} \times 25^{\prime \prime}$. Along the long axis of the array, the detectors are separated by approximately half a beamwidth (see Smith et al. 1991, 1994), while the two banks of detectors in the 20 channel system are separated by $28^{\prime \prime}$ at $100 \mu \mathrm{m}$ and $18^{\prime \prime}$ at $50 \mu \mathrm{m}$.

With the 8 channel system, the observations were made with a scanning mode (see Smith et al. 1991), where the array was scanned across the galaxy perpendicular to the array axis, resulting in 8 simultaneous azimuthal scans across the galaxy. The scan lengths ranged from $2{ }^{\prime} \cdot 3$ to 3.2 , and the data were binned at $2^{\prime \prime}$ intervals with a scan rate of $5^{\prime \prime} \mathrm{sec}^{-1}$. With the 20 channel system, the scanning method was used for NGC 3256, giving 10 parallel scans across the galaxy, while a nodding mode (see Smith et al. 1994) was used for four galaxies. In this method, the array was nodded on and off the galaxy at 10 second intervals. For NGC 891, data were taken at 2 overlapping array locations on the sky, shifting along the major axis of the array. For NGC 7331, three overlapping sets of data were obtained. Chopping was in the azimuthal direction, with amplitudes which varied from $3^{\prime}-6.5$. For both of these systems, the long axis of the array was aligned with the altitude axis of the telescope, causing the position angle of the array on the sky to vary during a long integration. The 
ranges in these rotation angles are given in Table 2. With the exception of NGC 1808 and NGC 6946, these rotation angles changed less than $12^{\circ}$ during the observations. For the NGC 7331 observations, the KAO 'freeze mode' was used, keeping the rotation angle steady to $\leq 1^{\circ}$.

For the April 22, 1988 observations of M 83 and for NGC 7331, we guided on the nucleus of the galaxy itself. For the rest, offset guiding was used, since the nuclei were too faint to guide on. The combination of offset guiding and diurnal motion causes the position of the center of the array on the sky to vary with time. To compensate for this effect, the offset was re-calculated and adjusted after each half sweep to keep the galaxy centered on the array.

To obtain a point source profile and to calibrate the data, we also observed at least one of the following objects during each flight: IRC +10216 , IRC +10420 , $\eta$ Carinae, Ceres, Vesta, Callisto, or Uranus. Details on these observations are also given in Table 2. Callisto, the asteroids, and Uranus all have angular diameters less than $4^{\prime \prime}$, so are clearly point sources. IRC +10216 , IRC +10420 , and $\eta$ Carinae have also been shown to be point sources at 50 and $100 \mu \mathrm{m}$ at this resolution (Lester, Harvey, and Joy 1986b; Harvey et al. 1991; Harvey, Hoffmann, and Campbell 1978).

\section{The Data}

After noise spike removal, the scan data were offset-adjusted, filtered, flatfielded, and co-added as in Smith et al. (1991). On the May 23, 1990 flight, strong correlated noise between the detectors was seen. To eliminate this noise, for those observations we subtracted the signal in channel 1 from that in the rest of the detectors before co-addition.

The final summed scans are shown in Figure 1. These plots show that the galaxies are all relatively compact compared to the instrumental profile. To make this comparison more directly, in Figure 2 we plot the galaxy and corresponding 
calibration data for the channel with the strongest signal. These plots show that M 83 is extended with respect to the point source, while NGC 253 and NGC 6946 appear marginally resolved. The rest are unresolved. To make this assessment more quantitative, we determined the rms deviation of each galaxy profile from the point source profile, within the range where the galaxy data remained above zero. Only for M 83 is the rms greater than $3 \sigma$. For the rest, we consider our derived source sizes to be upper limits.

To get an estimate of the source size in the cross-scan direction, we averaged the central $22^{\prime \prime}(100 \mu \mathrm{m})$ or $10^{\prime \prime}(50 \mu \mathrm{m})$ of the summed scans for each detector. These profiles along the long axis of the array are given in Figure 3. None of the galaxies are strongly resolved relative to the point source profiles. NGC 1097 and NGC 6946 appear marginally resolved, with possible low-level extended emission in the end channels, while the rest are unresolved.

The nod data were co-added as in Smith et al. (1994), and the summed data profiles are shown in Figure 4. These plots show that, if the far-infrared peak was well-centered on the first bank of detectors, then NGC 660, M 83, and NGC 7331 are resolved along the long axis of the array, while NGC 891 is unresolved. NGC 7331 is the most extended galaxy in this sample, showing a very broad flattopped profile with an approximate FWHM size of $165^{\prime \prime}$. The nod data for M 83 (Figure 4c) show a resolved central source with surrounding extended emission. To test whether the central peak is truly resolved above this plateau, we have re-plotted these data in Figure 4d with the end channels set to zero. In this figure, the central source appears only marginally extended, so we conservatively consider our derived size an upper limit.

All four galaxies in Figure 4 appear resolved along the minor axis of the array, if we are correctly centered with the infrared peak in the first bank of detectors. 
The flux observed in the second bank of detectors (lower panels in Figure 4a-e) is higher for the galaxies than for the point source profiles.

In Table 3, we give an estimate of the position of the far-infrared maxima in the sample galaxies. For the scan data, these are simply the location of the peak position for the detector with the strongest signal; we did not attempt to interpolate between detectors. For the M 83 and NGC 660 nod data, the positions given are the location of the detector with the strongest signal, and for NGC 891, we have averaged the positions of the two detectors in the first bank which have approximately equal flux densities. For NGC 7331, which does not have a strong far-infrared peak, we give the approximate center of the broad profile seen in the data from the first bank. Our absolute pointing accuracy is $10^{\prime \prime}$. For galaxies which are poorly centered on the array, there is an additional uncertainty in the location of the far-infrared peak. In Table 1, we provide the best available published measurements of the positions of the nuclei of these galaxies, from either radio or optical data. The far-infrared positions in Table 3 are all consistent with these nuclear positions within our uncertainties. Therefore, for the nod data shown in Figure 4, if the far-infrared distribution is symmetrical along the minor axis of the array with respect to the nucleus, then the galaxy is spatially resolved in this direction.

From the data plotted in Figures 2, 3, and 4, we have made estimates of the intrinsic sizes of the central far-infrared sources in these galaxies using a simple Gaussian deconvolution. These results are given in Table 3. The FWHM sizes range from $\leq 13^{\prime \prime}$ to $165^{\prime \prime}$ ( $\leq 220 \mathrm{pc}-12 \mathrm{kpc}$ ). For the nod data, the minor axis sizes were determined assuming symmetry about the nucleus and so are very uncertain.

The calibrated flux densities are given in Table 3. We estimate that these measurements are accurate to $30 \%$. For these calibrations, we assumed that 
Uranus is a blackbody of $58.5 \mathrm{~K}$ (Stier et al. 1978) with a diameter of 50,800 km, and Callisto is a blackbody of temperature of $147 \mathrm{~K}$ (Campbell et al. 1989) and diameter $5020 \mathrm{~km}$ (Jones and Morrison 1974). For Ceres and Vesta, we used a standard thermal model of the far-infrared emission from asteroids (Lebofsky et al. 1986), along with the IRAS diameters of $848 \mathrm{~km}$ and $468 \mathrm{~km}$ and albedos of 0.11 and 0.42, respectively (IRAS Minor Planet Survey 1992).

Determining absolute flux densities using the evolved stars $\eta$ Carinae, IRC +10420 , and IRC +10216 requires cross-calibration due to the possibility of farinfrared variability. To calibrate the March 1992 M 83 data, we use an $\eta$ Carinae $100 \mu \mathrm{m}$ flux density of $5200 \mathrm{Jy}$ (Harvey et al. 1978), since we confirmed earlier in the same flight series that the $100 \mu \mathrm{m}$ flux density had not changed (Smith et al. 1994). We cross-correlated the August 1990 IRC +10420 data using Hygeia observations from a different flight during the same flight series, and find values consistent with the Harvey and Wilking (1982) flux densities of $\mathrm{F}(50)=930 \mathrm{Jy}$ and $\mathrm{F}(100)=240 \mathrm{Jy}$ (Smith, Harvey, and Lester 1995). Also, using the August 23, 1990 Vesta data to calibrate IRC +10420 gives a consistent value of $\mathrm{F}(50)=$ $941 \mathrm{Jy}$. For the August 1994 observations we have no independent calibration of IRC +10420 , however, observations of Uranus during the same flight series give a calibration conversion consistent with that obtained using IRC +10420 with the flux densities given in Harvey and Wilking (1982). For the April 1988 M 83 data, we used the PSC color-corrected $100 \mu \mathrm{m}$ flux density of IRC +10216 of 862 Jy. This agrees within $15 \%$ with our independent calibration of $\mathrm{F}(100)=$ 759 Jy for IRC +10216 based on Uranus observations using unpublished April 1989 data. Table 3 shows that the peak M 83 brightnesses from the two different observations agree well.

In Table 3, we provide both a peak flux density and a total flux density. In some cases, because the galaxy was not well-centered on the array, the peak flux 
density does not equal the total flux density although the galaxy is unresolved. These incidences are noted in Table 3. In Table 3, we also give the total IRAS flux densities for the galaxies, obtained either from Rice et al. (1988) or IRAS Addscans. At $50 \mu \mathrm{m}$, we estimate total flux densities from the IRAS 25 and $60 \mu \mathrm{m}$ data assuming a $\lambda^{-1}$ emissivity law. The total flux densities from the KAO agree with the total IRAS flux densities within the uncertainties for all of the galaxies except for NGC 891, M 83, and NGC 6946. For these galaxies, our KAO observations are clearly missing extended emission. For the rest of the galaxies, less than $30 \%$ of the total far-infrared emission arises outside of the region observed by the $\mathrm{KAO}$.

Three of the galaxies in our sample were previously observed at 50 or 100 $\mu \mathrm{m}$ in a small beam (Rickard and Harvey 1984; Engargiola 1991). Our new peak brightness measurements are consistent with these data within the uncertainties (see Table 3).

\section{Discussion of Individual Galaxies}

Comparing the far-infrared morphology of individual galaxies with the observed distribution of star formation regions and molecular gas is useful in determining the origin of extragalactic far-infrared radiation. In this section, we discuss each galaxy in our sample individually, summarizing the available data at other wavelengths, and noting how the far-infrared distribution compares with that of the other components of the galaxy.

\section{1. $N G C 253$}

NGC 253 is a highly inclined spiral galaxy with a weak bar (Pence 1980; Scoville et al. 1988). Near-infrared spectroscopy of the nuclear region of NGC 253 reveals an obscured central starburst (Wynn-Williams et al. 1979; Rieke et al. 1980; Beck and Beckwith 1984; Rieke, Lebofsky, and Walker 1988). The CO $(1-0)$ and $(2-1)$ distributions are centrally peaked (Scoville et al. 1988; 
Mauersberger et al. 1995), and the CO $(2-1)$ size is $\sim 40^{\prime \prime} \times 10^{\prime \prime} \mathrm{FWHM}$ elongated east-west (Wall et al. 1991; Mauersberger et al. 1995). Denser gas appears to be more centrally concentrated; the ${ }^{13} \mathrm{CO}(3-2)$ source FWHM is $\leq 15^{\prime \prime}$ (Wall et al. 1991). The $1.3 \mathrm{~mm}$ and $3 \mathrm{~mm}$ FWHM sizes are $16^{\prime \prime} \times 11^{\prime \prime}$ and $10^{\prime \prime} \times 4^{\prime \prime}$, respectively (Krügel et al. 1990; Carlstrom et al. 1990). At $10-30$

$\mu \mathrm{m}$, the FWHM size is $7^{\prime \prime} \times \leq 4^{\prime \prime}$ (Telesco, Dressel, and Wolstencroft 1993). Our far-infrared source size limits $\left(\leq 16^{\prime \prime} \times 14^{\prime \prime}\right.$ and $\leq 21^{\prime \prime} \times \leq 31^{\prime \prime}$ at 50 and $100 \mu \mathrm{m}$, respectively), show that the far-infrared emitting region, particularly at $50 \mu \mathrm{m}$, is more compact that the $\mathrm{CO}(2-1)$ emission, but consistent with the sizes at the other wavelengths.

Low-level extended far-infrared disk emission is observed in NGC 253 out a diameter of 26.5 at $60 \mu \mathrm{m}$ (Rice et al. 1988). Our KAO observations show that this extended emission is a relatively small percentage, $\leq 30 \%$, of the total farinfrared emission from the galaxy, with the majority of the radiation arising from the central unresolved source.

\section{2. $N G C 660$}

NGC 660 is a peculiar barred spiral galaxy with a possible polar ring (Young, Kleinmann, and Allen 1988). It has an 'N'-type optical spectrum (Keel 1984), however, $\mathrm{H} \alpha+[\mathrm{N}$ II] mapping indicates significant massive star formation over an extended region in the center of the galaxy (Young et al. 1988). The CO distribution is extended northwest to southeast along the bar, with a FWHM size of $\sim 22^{\prime \prime} \times 35^{\prime \prime}$ (van Driel et al. 1995). We find a FWHM size of the $50 \mu \mathrm{m}$ emitting region of $22^{\prime \prime} \times \sim 46^{\prime \prime}(1.0 \mathrm{kpc} \times 2.1 \mathrm{kpc})$, similar to the CO size.

\section{3. $N G C 891$}

NGC 891 is an edge-on Sb galaxy thought to be very similar to the Milky Way. The CO $(1-0)$ distribution is centrally peaked with a surrounding molecular ring (Scoville et al. 1993), while the HI map shows a central depression 
(Rupen 1991). In the IRAS data, NGC 891 was resolved along its major axis but unresolved along its minor axis (Wainscoat, de Jong, and Wesselius 1987). Along the major axis, the far-infrared traces the radio continuum and CO (1 - 0) emission well (Wainscoat et al. 1987). With the KAO, we have further constrained the $100 \mu \mathrm{m}$ size of NGC 891 along its minor axis to be $\leq 37^{\prime \prime}$. NGC 891 is extended along its major axis in the far-infrared, with $\sim 25 \%$ of the total $100 \mu \mathrm{m}$ emission coming from the central $31^{\prime \prime} \times 41^{\prime \prime}$.

\section{4. $N G C 1097$}

NGC 1097 is a beautiful barred spiral with a LINER nucleus surrounded by a ring of star formation of diameter $15^{\prime \prime}-20^{\prime \prime}$ (Osmer, Smith, and Weedman 1974; Meaburn et al. 1981). This ring is present in radio continuum and $\mathrm{H} \alpha$ maps (Hummel et al. 1987), at $10 \mu \mathrm{m}$ (Telesco and Gatley 1981; Telesco et al. 1993), and in the CO ( $\mathrm{J}=1-0)$ line (Gérin et al. 1987). Our $100 \mu \mathrm{m}$ data show a central unresolved core with a source size of $\leq 36^{\prime \prime} \times \leq 41^{\prime \prime}$, consistent with the size of the star forming ring.

\section{5. $N G C 1808$}

The circumnuclear region of NGC 1808 contains several blue 'hot spot' H II regions (Morgan 1958; Sérsic and Pastoriza 1965; Véron-Cetty and Véron 1985; Phillips 1993). The optical spectrum of the nucleus itself was classified as that of a Seyfert galaxy by Véron-Cetty and Véron (1985), but as indicative of OB stars by Phillips (1993). The central star formation complex has a size of $10^{\prime \prime} \times 20^{\prime \prime}$, extended southeast to northwest (Véron-Cetty and Véron 1985). Outflow from this central area is indicated both by peculiar dust filaments extending out of this region perpendicular to the plane of the galaxy (Burbidge and Burbidge 1968; Véron-Cetty and Véron 1985) and by velocity measurements of the ionized gas (Phillips 1993). Our $100 \mu \mathrm{m}$ source size limit of $\leq 29^{\prime \prime} \times \leq 34^{\prime \prime}$ is consistent with that of this star forming region. 


\section{6. $N G C 3256$}

NGC 3256, a peculiar galaxy with two long tidal tails (Sandage and Brucato 1979; Joseph and Wright 1985), is likely the remnant of the merger between two spiral galaxies (Graham et al. 1984). Ultraviolet, optical, and near-infrared spectroscopy of this galaxy indicate that it is undergoing a strong central starburst (Kinney et al. 1993; Storchi-Bergmann, Kinney, and Challis 1995; Kawara, Nishida, and Gregory 1987; Doyon, Joseph, and Wright 1994). Br $\gamma$ emission is detected over over a $27^{\prime \prime} \times 16^{\prime \prime}$ region, with a FWHM size of $8^{\prime \prime} \times 5^{\prime \prime}$ (Moorwood and Oliva 1994a). Emission at $10 \mu \mathrm{m}$ is seen to a radius of $10^{\prime \prime}$, with a FWHM size of $9^{\prime \prime} \times 7^{\prime \prime}$ (Graham et al. 1987). NGC 3256 has been mapped in the CO $(2-1)$ line by several groups (Sargent, Sanders, and Phillips 1989; Casoli et al. 1991; Aalto et al. 1991). Casoli et al. (1991) determine an intrinsic FWHM size of $25^{\prime \prime} \times 11^{\prime \prime}$, while Aalto et al. (1991) find a smaller FWHM size of $11^{\prime \prime} \times$ $6^{\prime \prime}$. Our far-infrared source size limit of $\leq 24^{\prime \prime} \times \leq 22^{\prime \prime}$ is consistent with these measurements.

\section{7. $M 83$}

The well-known barred spiral galaxy M 83 is undergoing rapid star formation in and around the nucleus, at the ends of the bar, and on the spiral arms (Talbot, Jensen, and Dufour 1979; Ondrechen 1985; Bohlin et al. 1983; Gallais et al. 1991). The HI distribution has a $2^{\prime}$ diameter central depression (Allen, Atherton, and Tilanus 1986), while the CO $(1-0)$ distribution is centrally peaked and traces the optical bar (Handa et al. 1990).

The IRAS data on M 83 show that it is quite extended in the far-infrared; Rice et al. (1988) give a $60 \mu \mathrm{m}$ size (to $25 \mathrm{mJy} \operatorname{arcmin}^{-2}$ ) of $15.6^{\prime}$. Marston (1989) deconvolved IRAS pointed observations data on M 83, and revealed a possible far-infrared bar. Our KAO data resolves the central $100 \mu \mathrm{m}$ source, giving a FWHM of $20^{\prime \prime} \times \leq 26^{\prime \prime}$. Thus the far-infrared emission from this central 
source has a size consistent with that of the central CO source (Handa et al. 1990). Approximately $20 \%$ of the total $100 \mu$ m emission from this galaxy arises from this central source.

\subsection{The Circinus Galaxy}

The Circinus galaxy (Freeman et al. 1977) is a large, nearby spiral galaxy with a compact nuclear radio source, optical and infrared spectra indicative of non-thermal activity, and nuclear $\mathrm{H}_{2} \mathrm{O}$ maser emission (Moorwood and Glass 1984; Moorwood and Oliva 1988; Oliva et al. 1994). A strong $9.7 \mu \mathrm{m}$ silicate absorption feature is seen towards the nucleus (Moorwood and Glass 1984). $\mathrm{Br} \gamma$ imaging shows a compact central source plus two extranuclear HII region complexes at a radius of $5^{\prime \prime}$ (Moorwood and Oliva 1994b). Circinus is unresolved in the IRAS CPC data with a resolution of $\sim 1^{\prime}$ (Ghosh et al. 1992; van Driel et

al. 1993). Our observations further confine the region of far-infrared emission to a $\leq 17^{\prime \prime}-\leq 28^{\prime \prime}(\leq 330-\leq 540 \mathrm{pc})$ diameter area.

\subsection{NGC 6946}

NGC 6946 is an Scd galaxy with a moderately active starburst nucleus (Keel 1984). The molecular gas distribution in NGC 6946 is centrally peaked (Young and Scoville 1982) and elongated in a bar or spiral arm pattern (Ball et al. 1985; Weliachew et al. 1988; Ishizuki et al. 1990; Regan and Vogel 1995), while the atomic gas distribution has a central depression (Rogstad, Shostak, and Rots 1973).

There have been several studies of the far-infrared morphology of NGC 6946 . Low resolution IRAS maps (Rice et al. 1988) give a $60 \mu \mathrm{m}$ radius of $13.3^{\prime}$ to $\mu_{60}$ $=25 \mathrm{mJy} \operatorname{arcmin}^{-2}$. With the KAO, NGC 6946 was mapped at 120 and $170 \mu \mathrm{m}$ at $49^{\prime \prime}$ resolution (Smith, Harper, and Loewenstein 1984), at 100 and $160 \mu \mathrm{m}$ at $45^{\prime \prime}$ resolution, and at $200 \mu \mathrm{m}$ at $56^{\prime \prime}$ resolution (Engargiola 1991). These maps show a central emission peak, an extended disk, and some evidence for spiral 
structure in the disk emission. Our higher resolution data set a limit of $\leq 24^{\prime \prime}$ $\times \leq 34^{\prime \prime}(\leq 525 \mathrm{pc} \times \leq 710 \mathrm{pc}) \mathrm{FWHM}$ on the size of the central $100 \mu \mathrm{m}$ source, consistent with the size of the central CO (Ishizuki et al. 1990) and H $\alpha$ (Roy and Belley 1993) source. We find that $20 \%$ of the total $100 \mu \mathrm{m}$ flux density of NGC 6946 comes from this central source.

4.10. $N G C 7331$

NGC 7331 is an Sb galaxy with a prominent bulge (Boroson et al. 1981) and a weak LINER-like nuclear optical emission line spectrum (Keel 1983a). Narrowband $\mathrm{H} \alpha+[\mathrm{N} \mathrm{II}]$ imaging of this galaxy (Keel 1983b; Pogge 1989) reveals a stellarlike nucleus surrounded by diffuse ionized gas within the bulge and bright HII regions in the spiral arms. The diffuse bulge emission has line ratios similar to those of the nucleus (Keel 1983b). Radio continuum maps show a compact central source and faint diffuse bulge emission, in addition to prominent spiral arms (Cowan, Romanishin, and Branch 1994). HI data show a central depression in the atomic gas distribution (Bosma 1981). Young and Scoville (1982) observed the major axis of NGC 7331 in the CO $(1-0)$ line at $45^{\prime \prime}$ resolution and found a pronounced central hole; their central position, however, was offset $19^{\prime \prime}$ from the optical center. Subsequently, NGC 7331 was partially mapped in the CO (1 0 ) and $(2-1)$ lines at $23^{\prime \prime}$ and $12^{\prime \prime}$ resolution, respectively (Braine et al. 1993; Braine 1995, private communication). These data show a relatively constant CO $(1-0)$ surface brightness over the central $1^{\prime} .3 \times 0.5$, with a possible $30 \%$ dip near the nucleus. In the higher resolution CO $(2-1)$ data, this dip is more pronounced, with a spiral arm/nucleus contrast of approximately 3. Near-infrared data show redder colors at the spiral arms than at the bulge, also suggesting a central dip in the distribution of dust and gas (Telesco, Gatley, and Stewart $1982)$. 
Our $100 \mu \mathrm{m}$ major axis profile for NGC 7331 has a fairly flat-topped FIR distribution with a FWHM of approximately $165^{\prime \prime}$, without a pronounced central depression or a bright compact nuclear source. This far-infrared plateau covers not just the bulge area, but also crosses the spiral arms at a radius of $\sim 50^{\prime \prime}$. We have also resolved the minor axis emission, with a FWHM size of $\sim 42^{\prime \prime}$. This distribution is consistent with the lower resolution $100 \mu \mathrm{m}$ IRAS CPC data of van Driel et al. (1993).

In Figure 5, we compare our $100 \mu \mathrm{m}$ profile with the corresponding CO (1 0) data from Braine et al. (1993; 1995, private communication), obtained with a similar beamsize. Within the uncertainties, these distributions agree; our data do not rule out a $30 \%$ depression at $100 \mu \mathrm{m}$ near the center of NGC 7331. In Figure 5, we have also plotted the major axis $\mathrm{H} \alpha+[\mathrm{N} \mathrm{II}]$ profile for NGC 7331, derived from the Pogge (1989) image. In this figure, we plot an unsmoothed cut across the image at the location of our array, to identify the locations of the spiral arms and the bright compact central source. In addition, we plot the $\mathrm{H} \alpha+[\mathrm{N} \mathrm{II}]$ profile after smoothing the image to the same resolution as our KAO data. Since the emission in the nuclear and bulge regions of this image is dominated by [N II] (Keel 1983a, 1983b) and may not be due to OB star ionization, to approximate the distribution of massive young stars in NGC 7331 we have made a third cut across the image, after removing this central emission and smoothing. As this figure shows, within our uncertainties the $100 \mu \mathrm{m}$ distribution is consistent with both the original smoothed $\mathrm{H} \alpha+[\mathrm{N}$ II] image and the smoothed image with the bulge light removed. Higher $\mathrm{S} / \mathrm{N}$ and resolution are needed to unambiguously distinguish between these two distributions. NGC 7331 is highly inclined $\left(75^{\circ}\right.$; Bosma 1978), and the spiral arm HII regions along the minor axis are partially contained within the central beam.

4.11. $N G C 7552$ 
The barred spiral galaxy NGC 7552 has an 'amorphous' nucleus (Sérsic and Pastoriza 1965), with an emission line optical spectrum which has been classified as HII region-like (Ward et al. 1980) or as a weak LINER (Durret and Bergeron $1987,1988)$. Although diffuse ionized gas is observed to a radius of $1^{\prime}$, the bright

central region is relatively compact in $\mathrm{H} \alpha+[\mathrm{N} \mathrm{II}]$, with a FWHM size of $\sim 10^{\prime \prime}$ (Durret and Bergeron 1987). Radio and Br $\gamma$ maps of NGC 7552 reveal a nuclear starburst ring of diameter $7^{\prime \prime} \times 9^{\prime \prime}$ (Forbes et al. 1994; Forbes, Kotilainen, and Moorwood 1994). Our KAO data of this galaxy indicate that the far-infrared emission is arising from an unresolved central source of size $\leq 22^{\prime \prime} \times \leq 24^{\prime \prime}$, consistent with the size of this ring.

\section{Previously Published KAO Data}

To investigate the nature of far-infrared emission from galaxies, it is important to make comparisons between different galaxies as well as studies of individual galaxies. We now have a large enough sample of galaxies with high resolution KAO data available to make this possible.

In this section we describe and tabulate previously published KAO data on 11 additional galaxies. These galaxies were observed by the University of Texas KAO group using either one of the two detector systems described in Section 2, or a previous generation instrument. Since these data were obtained with either a scanning mode or with a multi-channel system, information about the spatial extent of the central far-infrared emission is available. These additional galaxies are listed in Table 4, along with their nuclear positions, distances, morphological types, blue magnitudes, diameters, and spectral types. We also include in Table 4 the galaxies M 31 and M 81, which are close enough that their bulge regions are resolved by IRAS.

In Table 5, we summarize the KAO results for the galaxies in Table 4, giving the wavelength of the observations, the average position angle, the source size, the 
observed flux density (both peak and total, when available), and the literature reference. We also include the total flux densities from IRAS, and the Rickard and Harvey (1984) or other published small aperture flux densities.

For all 24 galaxies in the combined sample, in Table 6 we tabulate the 50 and $100 \mu \mathrm{m}$ flux densities that we use in the following discussion, along with the measured far-infrared source sizes in arcseconds and the physical area $\Omega$ in $\mathrm{kpc}^{2}$. Ideally, we would like to compare the far-infrared emission from the same area within each galaxy, the inner $\sim 1 \mathrm{kpc}^{2}$. Since the galaxies are not all at the same distance, however, this physical area varies from galaxy to galaxy. Furthermore, some of the galaxies are so distant that the far-infrared size of the entire galaxy is used.

In Table 6, we also list the average dust temperature and $100 \mu \mathrm{m}$ optical depth in the observed regions, obtained from the $50 \mu \mathrm{m} / 100 \mu \mathrm{m}$ flux ratios assuming a $\lambda^{-1}$ emissivity law. For galaxies without $50 \mu \mathrm{m}$ measurements, we use the global IRAS $60 \mu \mathrm{m} / 100 \mu \mathrm{m}$ ratio to determine a dust temperature and therefore the optical depth. In these cases, if $\mathrm{T}_{d}$ (bulge) $>\mathrm{T}_{d}$ (global) then these estimates of $\tau_{100}$ are upper limits. We also include the IRAS M 31 bulge flux densities for a $4^{\prime}$ aperture (Soifer et al. 1986) in this table. In addition, we provide IRAS 60 and $100 \mu \mathrm{m}$ measurements for the bulge of M 81 in a $105^{\prime \prime}$ aperture obtained from the IRAS HiRes images (see Table 6).

\section{Additional Data}

In Tables 7.1, 7.2, and 8, we provide additional information about these galaxies. When possible, we have obtained $\mathrm{H} \alpha+[\mathrm{N}$ II] fluxes for the regions observed by the KAO (Table 7.1). The beamsizes for the $\mathrm{H} \alpha+[\mathrm{N} \mathrm{II}]$ measurements are also given in Table 7.1. For several of the galaxies in our sample, we acquired copies of published $\mathrm{H} \alpha+[\mathrm{N}$ II] images, from which we have extracted aperture measurements. For the other galaxies, we obtained fluxes directly from the literature. In 
these cases, the beamsizes are not always perfectly matched with the KAO beam. However, the optical apertures are generally larger than those of the far-infrared, and inspection of the published $\mathrm{H} \alpha+[\mathrm{N} \mathrm{II}]$ images shows that most of the emission comes from the central far-infrared source. For most of these galaxies, the filters used for the narrow-band optical observations were wide enough that they contain both $\mathrm{H} \alpha$ and the nearby $\lambda 6584 \AA$ [N II] line. Exceptions are noted in Table 7.1 .

We also give published $\operatorname{Br} \gamma$ fluxes in Table 7.1; these are only sometimes useful, however, because often the only available $\operatorname{Br} \gamma$ measurements are in apertures significantly smaller than our KAO beam. In Table 7.1, we also list published $\mathrm{CO}(1-0)$ measurements obtained in beam sizes similar to the KAO beam. When necessary, these have been corrected for the telescope aperture efficiency to a main beam temperature scale $\left(\mathrm{T}_{M B}\right)$, suitable for extended sources that fill the beam. In the following comparison with the far-infrared data, we use the first tabulated value of these parameters for each galaxy.

For many of these galaxies, spectroscopic measurements of the Balmer decrement $\mathrm{F}(\mathrm{H} \alpha) / \mathrm{F}(\mathrm{H} \beta)$ are also available. These are given in Table 7.2, along with their beamsizes, which are generally much smaller than the KAO beamsize. In addition, we also compile published values of $\mathrm{F}(\mathrm{H} \alpha) / \mathrm{F}(\mathrm{Br} \gamma)$ in Table 7.2.

We have also gathered BVH data for these bulges at aperture sizes similar to the KAO beam, using the NASA Extragalactic Database (NED; 1995) and Longo and deVaucouleurs $(1983,1988)$. From these data, we have estimated magnitudes within our KAO beams assuming a linear log(aperture)-magnitude relationship. If only one measurement at a given wavelength is available, it is used in the subsequent analysis if the aperture is within $30 \%$ of the KAO beam. In Table 8, we tabulate the interpolated magnitudes we use in this study. Finally, in Table 9, we list some derived quantities for the observed regions of these 
galaxies: the far-infrared, $\mathrm{H} \alpha+[\mathrm{N} \mathrm{II}]$, and blue luminosities, along with the ratios $\mathrm{L}(\mathrm{FIR}) / \mathrm{L}(\mathrm{H} \alpha+[\mathrm{N} \mathrm{II}]), \mathrm{L}(\mathrm{FIR}) / \mathrm{L}(\mathrm{B}), \mathrm{L}(\mathrm{FIR}) / \Omega$, and $\mathrm{L}(\mathrm{B}) / \Omega$.

\section{Discussion}

\subsection{Extinction}

To determine the origin of the far-infrared emission, one must first calculate the extinction towards the region in question. Our data provides one such estimate, the $100 \mu \mathrm{m}$ optical depth (Table 6). In Figure 6, we compare these values with the integrated $\mathrm{CO}$ intensity $\mathrm{I}(\mathrm{CO})$, another measure of the optical depth. In this plot and in subsequent figures, the galaxies with optical or nearinfrared signatures of strong star formation and/or Seyfert activity within the KAO beam (see Tables 1 and 4, and Section 4) are plotted as asterisks, while the galaxies with more quiescent bulges are plotted as filled triangles. M 81, which has an extremely weak Seyfert nucleus, is considered a quiescent galaxy.

Figure 6 shows that $\mathrm{I}(\mathrm{CO})$ and $\tau_{100}$ are well-correlated, and the galaxies with starburst or Seyfert activity lie mostly in the upper righthand section of this plot, with higher gas and dust column densities. The line plotted in Figure 6 is the expected relationship if both $\mathrm{CO}$ and $\tau_{100}$ trace extinction, using $\mathrm{A}_{\mathrm{V}} / \tau_{100}$ $=750$ (Makinen et al. 1985) $\left(\lambda^{-1}\right.$ emissivity law) and assuming the standard Galactic $\mathrm{I}(\mathrm{CO}) / \mathrm{n}\left(\mathrm{H}_{2}\right)$ (Bloemen et al. 1986) and $\mathrm{N}(\mathrm{H}) / \mathrm{A}_{\mathrm{V}}$ (Bohlin, Savage, and Drake 1978) ratios. The data points in Figure 6 generally lie above this line, showing that the $\mathrm{CO}$ data imply optical depths a factor of $\sim 4$ higher than the far-infrared data on average. This discrepancy has been noted before in comparisons of the global $\mathrm{M}\left(\mathrm{H}_{2}\right)$ to $\mathrm{M}$ (dust) ratio (Young et al. 1989; Devereux and Young 1990) and in a comparison of single aperture far-infrared data with published CO data (Rickard and Harvey 1984). It may be due to a number of

effects. The $\mathrm{A}_{\mathrm{V}} / \tau_{100}$ ratio is poorly known and may vary significantly from location to location; published values range from 280 - 2600 (Harvey, Thronson, 
and Gatley 1980; Whitcomb et al. 1981; Hildebrand 1983; Makinen et al. 1981; Casey 1991). Also, extragalactic observations at $100 \mu \mathrm{m}$ may miss a significant amount of very cold dust (Eales, Wynn-Williams, and Duncan 1989; Kwan and Xie 1992). In addition, the $\mathrm{I}(\mathrm{CO}) / \mathrm{n}\left(\mathrm{H}_{2}\right)$ ratio may be enhanced in starburst nuclei (Maloney and Black 1988; Maloney 1990; Mauersberger et al. 1996a,b). Scatter is also introduced by the differing beamsizes, the lack of small beam 50 $\mu \mathrm{m}$ data for many galaxies, and the possibility of clumpiness of the interstellar medium within the KAO and CO beams.

Other methods of determining extinction are also quite uncertain, particularly the broadband colors. The B - V color (Table 8.2) does not correlate with $\mathrm{I}(\mathrm{CO})$; starburst and quiescent galaxies have similar colors. $\mathrm{B}-\mathrm{V}$ does not trace extinction well, because it is affected not only by dust but also the stellar population. Also, because the dust is likely mixed with the stars rather than in a foreground screen, the reddening relative to the extinction is reduced (Witt, Thronson, and Capuano 1992). For a few individual galaxies, detailed models of the optical and near-infrared colors which take into account geometric effects have recently been developed by a number of groups. For NGC 4736, extinctions derived from this method are consistent with those obtained from the CO $(1-0)$ line (Block et al. 1994).

Hydrogen emission line ratios may also underestimate extinction because of mixing. In Figure 7, we compare $\mathrm{I}(\mathrm{CO})$ with the $\mathrm{A}_{\mathrm{V}}$ derived from the Balmer decrement. For this calculation, we used the standard Galactic extinction curve and an intrinsic $\mathrm{F}(\mathrm{H} \alpha) / \mathrm{F}(\mathrm{H} \beta)=2.85$ (Osterbrock 1989), except for the data for the narrow line region of $\mathrm{M} 81$, where an intrinsic $\mathrm{F}(\mathrm{H} \alpha) / \mathrm{F}(\mathrm{H} \beta)=3.1$ was assumed (Filippenko and Sargent 1988). This plot shows little correlation, and the extinctions implied by the Balmer decrement are generally smaller than those given by $\mathrm{I}(\mathrm{CO})$. The $\mathrm{F}(\mathrm{H} \alpha) / \mathrm{F}(\mathrm{Br} \gamma)$ ratio is compared with $\mathrm{I}(\mathrm{CO})$ in Figure 
8. There is somewhat better agreement, but the hydrogen line ratio still gives lower extinction values. This may be due to a combination of underestimation by $\mathrm{F}(\mathrm{H} \alpha) / \mathrm{F}(\mathrm{Br} \gamma)$ due to mixing and overestimation by $\mathrm{I}(\mathrm{CO})$.

Other methods of determining extinction include comparisons of these hydrogen lines with the $\mathrm{Pa} \beta$ and $\mathrm{Br} \alpha$ lines, thermal radio continuum emission, submillimeter dust continuum, and mid-infrared absorption feature strengths. Unfortunately, these data are available for only a few of our sample galaxies. Therefore, in the following discussion, we will rely on the optical depths determined from the CO intensities and the $100 \mu \mathrm{m}$ optical depths, with the caveats noted above. The true optical depth probably lies between that implied by the $\mathrm{CO}$ data and that given by the far-infrared data.

\subsection{Star Formation}

Galaxies which are actively forming stars are known to have higher global farinfrared luminosities and higher ratios of far-infrared to blue luminosities than less active galaxies. The data tabulated in Table 9 show that this is also true for the central regions of galaxies. The bulges of the quiescent galaxies, particularly M 31 and M 81, stand out as having low far-infrared luminosities relative to their blue luminosities. In Figure 9, we have scaled these luminosities by the area $\Omega$ of the source, to compare average surface brightnesses. The starburst and Seyfert galaxies have higher far-infrared surface brightnesses than the more quiescent galaxies. Accounting for the fact that many of the starburst/Seyfert galaxies are unresolved with the KAO makes this difference more pronounced. In this

plot, the blue surface brightnesses for the quiescent and more active galaxies are similar, but these are underestimated for the unresolved galaxies.

In Figure 10, far-infrared surface brightnesses are compared to the $\mathrm{CO}$ $(1-0)$ intensities. A strong correlation is seen, with higher far-infrared surface brightnesses with increasing optical depth. $\mathrm{L}(\mathrm{FIR}) / \mathrm{L}(\mathrm{B})$ is also correlated with 
I(CO) (Figure 11). Starburst/Seyfert galaxies have higher L(FIR)/L(B) ratios than the quiescent bulges.

The $\mathrm{H} \alpha$ luminosity, after correction for extinction, is an approximate measure of the rate of star formation, if ionization is due to OB stars. In Figures 12 and 13, we plot the ratio $\mathrm{L}(\mathrm{FIR}) / \mathrm{L}(\mathrm{H} \alpha)$ against our extinction measures $\mathrm{I}(\mathrm{CO})$ and $\tau_{100}$. When necessary, we have corrected for [N II] emission by assuming $\mathrm{L}(\mathrm{H} \alpha)$ $=0.75 \mathrm{~L}(\mathrm{H} \alpha+[\mathrm{N} \mathrm{II}])$ for the galaxies with strong star formation within the KAO beam, and $\mathrm{L}(\mathrm{H} \alpha)=0.25 \mathrm{~L}(\mathrm{H} \alpha+[\mathrm{N} \mathrm{II}])$ for the LINER galaxies (see Jacoby et al. 1989; Smith et al. 1991). In contrast to Figure 11, where I(CO) is strongly correlated with $\mathrm{L}(\mathrm{FIR}) / \mathrm{L}(\mathrm{B})$, little correlation is found with $\mathrm{L}(\mathrm{FIR}) / \mathrm{L}(\mathrm{H} \alpha)$. The quiescent galaxies NGC 4736, NGC 3627, and NGC 7331 have L(FIR)/L(H $\alpha)$ ratios higher than those of most of the starburst galaxies but lower CO intensities and $100 \mu \mathrm{m}$ optical depths. In these figures, we plot curves corresponding to the expected relationships for dust heating by OB stars following a Salpeter IMF, using the assumptions given above for extinction and an intrinsic $\mathrm{L}(\mathrm{FIR}) / \mathrm{L}(\mathrm{H} \alpha)$ ratio of 55 (Devereux and Young 1989). For this calculation, we assume that the extincted region lies halfway through the molecular disk.

Although there is considerable uncertainty as to the exact relationship between extinction, CO, and $\tau_{100}$ (see Section 6.1), these curves give an approximate dividing line between galaxies in which dust heating is dominated by OB stars and those in which the older stellar population is important. In Figure 12, the quiescent galaxies tend to lie above the curve, with infrared excesses higher than that expected by dust heating by massive young stars. The infrared excesses in the bulges of NGC 4736 and NGC 3627 relative to their extinctions have been noted before by Smith et al. (1991, 1994), who conclude that dust heating is dominated by the non-ionizing stellar population. This same conclusion was 
reached for the bulge of M 31 by Soifer et al. (1986). The central regions of NGC 7331 and M 81 may also have major contributions by non-OB stars.

This analysis assumes that all of the observed $\mathrm{H} \alpha$ in these galaxies is due to ionization by OB stars, however, this is probably not the case for the bulges of the quiescent LINER-like galaxies. The ionization mechanism responsible for the LINER-like optical line ratios in the centers of galaxies is still unclear. Shock excitation (Heckman 1980; Bonatto et al. 1989), photoionization by an active nucleus (Ferland and Netzer 1983), photoionization of dense clouds by massive O stars (Filippenko and Terlevich 1992; Shields 1992), collisional excitation due to mass loss from giant stars (Burbidge and Burbidge 1962; Burbidge, Gould, and Pottash 1963), and ionization by hot horizontal-branch stars (Minkowski and Osterbrock 1959) have all been suggested as possible ionization mechanisms. If something other than OB stars is responsible for the ionization, then the infrared excesses in these bulges relative to the star formation rate is further enhanced. In the bulges of M 31 and NGC 4736, the optical line ratios have been found to be LINER-like across the bulge, not just in the nucleus (Ciardullo et al. 1988; Smith et al. 1994).

In Figure 12, the starburst galaxies tend to lie to the right of the extinction curve, therefore, there are sufficient OB stars in these galaxies to power the farinfrared luminosity, if the $\mathrm{CO}$ traces the extinction. The fact that many of the starburst galaxies lie far to the right in this plot may be due to an overestimation of the $\mathrm{H} \alpha$ extinction by the CO. In contrast to Figure 12, in Figure 13 many of the starburst galaxies lie to the left of the curve; this may be due to an underestimation of $\mathrm{A}_{\mathrm{V}}$ by $\tau_{100}$.

The peculiar galaxy NGC 1275 also lies above the extinction curve, in the regime where dust heating by older stars is expected to dominate. However, NGC 1275 may be a special case, being the dominant galaxy in the X-ray luminous 
galaxy cluster Perseus. The dust in this galaxy may be heated by hot intracluster gas in a cooling flow rather than star formation or the older stellar population (Lester et al. 1995). Therefore it is unique in this sample, and we do not discuss it further in this paper.

These plots show that, regardless of the precise relationship between $\mathrm{I}(\mathrm{CO})$, $\tau_{100}$, and $A_{V}$, the trends are clear; quiescent galaxies have higher far-infrared excesses compared to starburst galaxies when extinction corrections are taken into account.

In Figures 14 and 15, we compare $\mathrm{L}(\mathrm{FIR}) / \mathrm{L}(\mathrm{Br} \gamma)$ with $\mathrm{I}(\mathrm{CO})$ and $\tau_{100}$ for the galaxies for which beam-matched $\operatorname{Br} \gamma$ data are available. All of these galaxies are known to have strong star formation in the KAO beam, and, in general, they fit the expected relationship for OB star dust heating, with possible underestimation of the optical depth by $\tau_{100}$ and overestimation by $\mathrm{I}(\mathrm{CO})$. It is possible that an active galactic nucleus contributes significantly to dust heating in a few of these galaxies. AGN dust heating is characterized by a high infrared excess compared to OB star heating (DePoy, Becklin, and Geballe 1987; Prestwich et al. 1994). An infrared excess has been cited before for Arp 220, based on extinctions derived from $\mathrm{F}(\operatorname{Br} \alpha) / \mathrm{F}(\operatorname{Br} \gamma), \mathrm{F}(\operatorname{Br} \gamma) / \mathrm{F}(\operatorname{Pa} \gamma)$, and millimeter continuum data (DePoy et al. 1987; Scoville et al. 1991; Prestwich et al. 1994; Larkin et al. 1995; Armus et al. 1995). The Seyfert nucleus in NGC 1068 may also contribute to dust heating. Further investigations of the extinction in these galaxies are needed to confirm these results.

\subsection{Dust Heating by the Older Stellar Population}

In Figure 11, we found that the far-infrared to blue luminosity ratio increases with $\mathrm{I}(\mathrm{CO})$. The blue light originates from a combination of both young and old stars. A better measure of the bolometric luminosity of the older stellar population is the near-infrared $\mathrm{H}$ band, which is also less affected by extinction. We 
compare the far-infrared to H-band luminosity ratio with $\mathrm{I}(\mathrm{CO})$ in Figure 16. As with $\mathrm{L}(\mathrm{FIR}) / \mathrm{L}(\mathrm{B})$, there is a correlation with optical depth. Unlike the case with $\mathrm{L}(\mathrm{FIR}) / \mathrm{L}(\mathrm{H} \alpha)$, there is a clear distinction between starburst/Seyfert galaxies and quiescent galaxies in $\mathrm{L}(\mathrm{FIR}) / \mathrm{L}(\mathrm{B})$ and $\mathrm{L}(\mathrm{FIR}) / \mathrm{L}(\mathrm{H})$, with starburst/Seyferts having significantly higher values.

For comparison, we plot an extinction curve in this figure, using the same assumptions as above. These curves are normalized to $\mathrm{L}(\mathrm{FIR}) / \mathrm{L}(\mathrm{H}) \sim 2$ at $\mathrm{A}_{\mathrm{V}}=$ 0, and pass near NGC 4736, NGC 3627, and NGC 7331. For a population of K5 giants, this curve is consistent with a situation where $\sim 1 / 5$ th of the total stellar luminosity is absorbed by dust and re-emitted in the far-infrared. These curves do not intersect $M 31$ and M 81, implying that, if dust heating is dominated by non-ionizing stars in all the quiescent galaxies, then a higher proportion of the bolometric luminosity of the older stars is absorbed by dust and re-emitted in the far-infrared in NGC 4736, NGC 3267, and NGC 7331 than in M 81 and M 81. This difference may be caused by both a larger amount of interstellar dust available for absorption and re-emission, which is clearly the case, and also a difference in stellar populations. These three galaxies have younger stellar populations and therefore more energetic radiation fields than M 31 and M 81 (Pritchet 1977; Keel 1983c; Walker, Lebofsky, and Rieke 1988).

In Figure 16, the curve through NGC 4736 agrees with the data for some of the starburst/Seyfert galaxies, however, many lie above the curve. Therefore, the difference in $\mathrm{L}(\mathrm{FIR}) / \mathrm{L}(\mathrm{H})$ between starburst/Seyfert galaxies and quiescent galaxies cannot simply be explained by extinction, but instead must be due to differences in dust cross section and stellar population. The relative proportion of energy coming out of the galaxy in the far-infrared relative to the optical/near-infrared is higher in starburst/Seyfert galaxies. Therefore, 
$\mathrm{L}(\mathrm{FIR}) / \mathrm{L}$ (old stars) is higher for starburst/Seyfert galaxies than quiescent galaxies, while $\mathrm{L}(\mathrm{FIR}) / \mathrm{L}$ (young stars) is similar or lower (Section 6.2).

These results can be explained by an increasing contribution to dust heating from young stars as one moves to higher extinctions. For the galaxies without optical/near-infrared signatures of star formation, dust heating is dominated by the underlying older stellar population. For more active galaxies, OB stars become important. This can also be seen in Figure 17, where $\mathrm{L}(\mathrm{H} \alpha) / \mathrm{L}(\mathrm{H})$ is plotted against $\mathrm{I}(\mathrm{CO}) . \mathrm{L}(\mathrm{H} \alpha)$ measures the number of observed $\mathrm{OB}$ stars (or, as noted above, for M 31, M 81, NGC 1275, and the LINERS, is an upper limit), while $\mathrm{L}(\mathrm{H})$ traces the older stellar population. When the unusual galaxy NGC 1275 is excluded, a segregation is seen between the quiescent galaxies, concentrated in the lower left corner, and the starburst/Seyfert galaxies, which have higher $\mathrm{L}(\mathrm{H} \alpha) / \mathrm{L}(\mathrm{H})$ ratios. Thus, at larger column densities of gas and dust, the proportion of young stars relative to old stars is higher.

In these figures, the separation between the starbursts and the more quiescent LINER bulges is at approximately $\log \mathrm{I}(\mathrm{CO})=1.5$, which corresponds to a molecular gas surface mass density of $140 \mathrm{M}_{\odot} \mathrm{pc}^{-2}$, using the Bloemen et al. (1986) conversion. This is the critical density at which star formation occurs

in a gaseous disk with a rotational velocity of $150 \mathrm{~km} \mathrm{~s}^{-1}$ at a radius of 500 pc (Kennicutt 1989). Thus the lack of powerful star formation in the bulges of NGC 4736, NGC 3627, NGC 7331, M 31, and M 81 may be due to an average gas density lower than the critical density.

\section{Conclusions}

Using the KAO, we have observed the central regions of 11 galaxies in the farinfrared at high spatial resolution. For 10 out of these 11 galaxies, optical and near-infrared data indicate massive star formation in the inner regions. For these 10, the far-infrared sizes or size limits are consistent with those of the central star 
formation complexes. The remaining galaxy, NGC 7331, does not have observed star formation in its bulge. It does not have a bright central far-infrared source, but instead has a very extended far-infrared distribution with similar $100 \mu \mathrm{m}$ surface brightnesses for its bulge and inner spiral arms.

We compare the small-beam far-infrared data for these galaxies to optical, near-infrared, and millimeter data, increasing the galaxy sample to 24 by including published KAO data for 11 additional galaxies and the IRAS data for the bulges of $\mathrm{M} 31$ and $\mathrm{M} 81$. We find that $\mathrm{L}(\mathrm{FIR}) / \mathrm{L}(\mathrm{B})$ and $\mathrm{L}(\mathrm{FIR}) / \mathrm{L}(\mathrm{H})$ correlate strongly with CO $(1-0)$ intensity and $100 \mu \mathrm{m}$ optical depth, while $\mathrm{L}(\mathrm{FIR}) / \mathrm{L}(\mathrm{H} \alpha)$ does not. Galaxies with optical or near-infrared signatures of $\mathrm{OB}$ star formation in their central regions have higher values of $\mathrm{I}(\mathrm{CO})$ and $\tau_{100}$ than more quiescent galaxies, as well as higher far-infrared surface brightnesses and $\mathrm{L}(\mathrm{FIR}) / \mathrm{L}(\mathrm{B})$ and $\mathrm{L}(\mathrm{FIR}) / \mathrm{L}(\mathrm{H})$ ratios. The $\mathrm{L}(\mathrm{FIR}) / \mathrm{L}(\mathrm{H} \alpha)$ ratios for starburst galaxies are consistent with $\mathrm{OB}$ stars dominating dust heating, while more quiescent bulges have infrared excesses too high to be accounted for by young stars alone. Whether or not a galaxy bulge has strong star formation, and therefore far-infrared luminosities powered by OB stars, may be decided by a threshold effect; star forming galaxies have surface gas densities above the Kennicutt critical density, while quiescent galaxies have lower values.

We would like to thank J. Bergeron, J. Braine, F. Casoli, F. Durret, M. Gérin, R. Pogge, R. Rand, M. P. Véron, and W. Waller for providing copies of their data for our use. We would also like to thank Cecilia Colomé, James DiFrancesco, Marshall Joy, Chris Koresko, Dan Lester, and Cheng-Yue Zhang for their help in making these observations. In addition, we acknowledge the help of Greg Doppmann in calculating the asteroid fluxes. We are very grateful to the KAO staff for their support during these flights, and to Dan Lester for helpful advice throughout this project. We also thank Sue Madden for helpful comments on 
this manuscript. This work was supported by NASA grant NAG 2-67. B. J. S. also acknowledges the support of a National Research Council-JPL Research Associateship during the final stages of this project. This research has made use of the NASA/IPAC Extragalactic Database (NED) which is operated by the Jet Propulsion Laboratory, Caltech, under contract with the National Aeronautics and Space Administration. 


\section{REFERENCES}

Allen, R. J., Atherton, P. D., and Tilanus, R. P. J. 1986, Nature, 319, 296.

Aalto, S., Black, J. H., Booth, R. S., and Johansson, L. E. B. 1991a, A\&A, 247, 291.

Aalto, S., Black, J. H., Johansson, E. B., and Booth, R. S. 1991b, A\&A, 249, 323.

Antonucci, R. R. J., and Ulvestad, J. S. 1988, ApJ, 330, L97.

Armus, L., Heckman, T. M., and Miley, G. K. 1989, ApJ, 347, 727.

Armus, L., Neugebauer, G., Soifer, B. T., and Matthews, K. 1995, AJ, 110, 2610.

Aumann, H. H., Fowler, J. W., and Melnyk, M. 1990, AJ, 99, 1674.

Barbon, R., Cappellaro, E., Ciatti, F., Turatto, M., and Kowal, C. T. 1984, A\&AS, 58, 735 .

Beck, S. C., and Beckwith, S. V. 1984, MNRAS, 207, 671.

Beck, S. C., Turner, J. L., and Ho, P. T. P. 1986, ApJ, 309, 70.

Belley, J., and Roy, J.-R. 1992, ApJS, 78, 61.

Block, D. L., Witt, A. N., Grosbol, P., Stockton, A., and Moneti, A., 1994, A\&A, 288,383 .

Bloemen, J. B. G. L. et al. 1986, A\&A, 154, 25.

Bonatto, C., Bica, E., and Alloin, D. 1989, A\&A, 226, 23.

Bohlin, R. C., Corelli, R. H., Hill, J. K., Smith, A. M., and Stecher, T. P. 1983, ApJ, 274, L53.

Bohlin, R. C., Savage, B. D., and Drake, J. F. 1978, ApJ, 224, 132.

Booth, R. S. et al. 1989, A\&A, 216, 315.

Boroson, T. 1981, ApJS, 46, 177.

Bosma, A. 1978, Ph.D. Thesis, University of Groningen.

Bosma, A. 1981, AJ, 86, 1791.

Braine, J., Combes, F., Casoli, F., Dupraz, C., Gérin, M., Klein, U., Wielebinski, R., and Brouillet, N. 1993, A\&AS, 97, 887. 
Brock, D., Joy, M., Lester, D. F., Harvey, P. M., and Ellis, H. B., Jr. 1988, ApJ, $329,208$.

Burbidge, E. M., and Burbidge, G. R. 1962, ApJ, 135, 694.

Burbidge, G. R., Gould, R. J., and Pottasch, S. R. 1963, ApJ, 138, 945.

Buta, R. 1988, ApJS, 66, 233.

Canzian, B., Mundy, L. G., and Scoville, N. Z. 1988, ApJ, 333, 157.

Campbell, M. F., Lester, D. F., Harvey, P. M., and Joy, M. 1989, ApJ, 345, 298.

Carlstrom, J. E., Jackson, J., Ho, P. T. P., and Turner, J. L. 1990, in The Interstellar Medium in External Galaxies: Summaries of Contributed Papers, (NASA CP-3084), 337.

Casey, S. C. 1991, ApJ, 371, 183.

Casoli, F., Combes, F., Augarde, R., Figon, P., and Martin, J. M. 1989, A\&A, 224,31 .

Casoli, F., Combes, F., Dupraz, C., Gérin, M., Encrenaz, P. and Salez, M. 1988, A\&A, 192, L17.

Casoli, F., Dupraz, C., and Combes, F. 1992, A\&A, 264, 49.

Casoli, F., Dupraz, C., Combes, F., and Kazès, I. A\&A, 1991, 251, 1.

Caulet, A. et al. 1992, ApJ, 388, 301.

Ciardullo, R., Rubin, V. C., Jacoby, G. H., Ford, H. C., and Ford, W. K., Jr. 1988, ApJ, 95, 438.

Claussen, M. J., and Shahai, R. 1992, AJ, 103, 1134.

Clements, E. D. 1981, MNRAS, 197, 829.

Condon, J. J., Condon, M. A., Gisler, G., and Puschell, J. J. 1982, ApJ, 252, 102.

Corwin, H. G., Jr, 1991, Internal NED Report, 'Positions for Selected Extragalactic Objects in the NED Database'.

Cowan, J. J., Romanishin, W., and Branch, D. 1994, ApJ, 436, L139.

Coziol, R., Demers, S., Pena, M., and Barneoud, R. 1994, AJ, 108, 405. 
Crane, P. C., Dickel, J. R., and Cowan, J. J. 1992, ApJ, 390, L9.

Dahari, O. 1985, ApJS, 57, 643.

Dahlem, M., Aalto, S., Klein, U., Booth, R., Mebold, U., Wielebinski, R., and Lesch, H. 1990, A\&A, 240, 237.

Dame, T. M., Koper, E., Israel, F. P., and Thaddeus, P. 1993, ApJ, 418, 730.

de Vaucouleurs, G., and de Vaucouleurs, A. 1972, Mem. R. A. S., 77, 1.

de Vaucouleurs, G. 1975, in Stars and Stellar Systems, Vol. 9, Galaxies and the Universe, ed. A. Sandage, M. Sandage, and J. Kristian (Chicago: Univ. of Chicago Press), 309.

DePoy, D. L., Becklin, E. E., and Geballe, T. R. 1987, ApJ, 316, L63.

DePoy, D. L. 1996, New Extragalactic Perspectives in the New South Africa: Changing Perceptions of the Morphology, Dust Content, and Dust-Gas Ratios in Galaxies, ed. D. Block, in press.

Dettmar, R.-J. 1990, A\&A, 232, L15.

Devereux, N. A., and Young, J. S. 1990, ApJ, 359, 42.

Devereux, N. A., and Young, J. S. 1992, AJ, 103, 1536.

Devereux, N. A., and Young, J. S. 1993, AJ, 106, 948.

Devereux, N. A., and Scowen, P. A. 1994, AJ, 108, 1244.

Devereux, N. A., Price, R., Wells, L. A., and Duric, N. 1994, AJ, 108, 1667.

Devereux, N. A., Jacoby, G., and Ciardullo, R. 1995, AJ, 110, 1115.

Doyon, R., Joseph, R. D., and Wright, G. S. 1994, ApJ, 421, 101.

Dressel, L. L., and Condon, J. J. 1976, ApJS, 31, 187.

Deul, E. R. 1989, A\&A, 218, 78.

Durret, F., and Bergeron, J. 1987, A\&A, 173, 219.

Durret, F., and Bergeron, J. 1988, A\&AS, 75, 273.

Eales, S. A., Wynn-Williams, C. G., and Duncan, W. D. 1989, ApJ, 339, 859. 
Eckart, A., Cameron, M., Rothermel, H., Wild, W., Zinnecker, H., Rydbeck, G., Olberg, M., and Wiklind, T. 1990, ApJ, 363, 451.

Engargiola, G. 1991, ApJS, 76, 875.

Ferland, G. J., and Netzer, H. 1983, ApJ, 264, 105.

Filippenko, A. V., and Sargent, W. L. W. 1988, ApJ, 324, 134.

Filippenko, A. V., and Terlevich, R. 1992, 397, L79.

Fischer, J., Geballe, T. R., Smith, H. A., Simon, M., and Storey, J. W. V. 1987, ApJ, 320, 667 .

Fischer, J., Simon, M., Benson, J., and Solomon, P. M. 1983, ApJ, 273, L27.

Forbes, D. A., Norris, R. P., Williger, G. M., and Smith, R. C. 1994, AJ, 107, 984.

Forbes, D. A., Kotilainen, J. K., and Moorwood, A. F. M. 1994, ApJ, 433, L13.

Ford, H. C., Crane, P. C., Jacoby, G. H., Lawrie, D. G., and van der Hulst, J. M. 1985, ApJ, 293, 132.

Freeman, K. C., Karlsson, B., Lynga, G., Burrell, J. F., van Woerden, H., Goss, W. M., and Mebold, V. 1977, A\&A, 55, 445.

Gallais, P., Rouan, D., Lacombe, F., Tiphène, D., and Vauglin, I. 1991, A\&A, 243,309 .

Gallagher, J. S., Hunter, D. A., Gillett, F. C., and Rice, W. L. 1991, ApJ, 371, 142.

Garman, L. E., and Young, J. S. 1986, A\&A, 154, 8.

Gérin, M., Nakai, N., and Combes, F. 1988, A\&A, 203, 44.

Gérin, M., Casoli, F., and Combes, F. 1991, A\&A, 251, 32.

Ghosh, S. K., Bisht, R. S., Iyengar, K. V. K., Rengarajan, T. N., Tandon, S. N., and Verma, R. P. 1992, ApJ, 391, 111.

Goad, J., and Gallagher, J. S., Jr. 1985, ApJ, 297, 98.

Graham, J. R. 1979, ApJ, 232, 60. 
Graham, J. R., Wright, G. S., Meikle, W. P. S., Joseph, R. D., and Bode, M. F. 1984, Nature, 310, 213.

Graham, J. R., Wright, G. S., Joseph, R. D., Frogel, J. A., Phillips, M. M., and Meikle, W. P. S. 1987, in Star Formation in Galaxies, ed. C. J. Lonsdale Persson (NASA CP-2466), 517.

Hall, D. N. B., Kleinmann, S. G., Scoville, N. Z., and Ridgway, S. T. 1981, ApJ, 248,898 .

Handa, T., Nakai, N., Sofue, Y., Hayashi, M., and Fujimoto, M. 1990, PASJ, 42, 1.

Harper, D. A., and Low, F. J. 1973, ApJ, 182, L89.

Harvey, P. M., and Wilking, B. A. 1982, PASP, 94, 285.

Harvey, P. M., Hoffmann, W. F., and Campbell, M. F. 1978, 70, 165.

Harvey, P. M., Lester, D. F., Brock, D., and Joy, M. 1991, ApJ, 368, 558.

Harvey, P. M., Thronson, H. A., and Gatley, I. 1980, ApJ, 235, 894.

Heckman, T. M., Balick, B., and Crane, P. C. 1980, A\&AS, 40, 295.

Heckman, T. M. 1980, A\&A, 87, 152.

Helfer, T. T., and Blitz, L. 1993, ApJ, 419, 86.

Helou, G. 1986, ApJ, 311, L33.

Hildebrand, R. H. 1983, QJRAS, 24, 267.

Ho, P. T. P., Beck, S. C., and Turner, J. L. 1990, ApJ, 349, 57.

Ho, L. C., Filippenko, A. V., and Sargent, W. L. W. 1995, ApJS, 98, 477.

Hummel, E., van der Hulst, J. M., and Keel, W. C. 1987, A\&A, 172, 32.

Humphreys, R. M., and Aaronson, M. 1987, AJ, 94, 1156.

Hutchings, J. B., Neff, S. G., Stanford, S. A., Lo, E., and Unger, S. W. 1990, AJ, 100,60 . 
IRAS Catalogs and Atlases: Explanatory Supplement 1988, ed. C. A. Beichman, G. Neugebauer, H. J. Habing, P. E. Clegg, and T. J. Chester (Washington DC: GPO).

IRAS Minor Planet Survey, 1992, edited by Tedesco, E. F. (Phillips Laboratory Technical Report No. PL-TR-92-2049, Hanscom Air Force Base, MA).

Israel, F. P., van Dishoeck, E. F., Baas, F., Koornneef, J., Black, J. H., and de Graauw, T. 1990, A\&A, 227, 342.

Jacoby, G. H., Ford, H., and Ciardullo, R. 1985, ApJ, 290, 136.

Joseph, R. D. and Wright, G. S. 1985, MNRAS, 214, 87.

Joy, M., Lester, D. F., and Harvey, P. M. 1987, ApJ, 319, 314.

Joy, M., Lester, D. F., Harvey, P. M., and Ellis, H. B. 1988, ApJ, 326, 662.

Joy, M., Lester, D. F., Harvey, P. M., and Frueh, M. 1986, ApJ, 307, 110.

Joy, M., Lester, D. F., Harvey, P. M., Telesco, C. M., and Decher, R. 1989, ApJ, $339,100$.

Kawara, K., Nishida, M., and Gregory, B. 1987, ApJ, 321, L35.

Keel, W. C. 1983a, ApJS, 52, 229.

Keel, W. C. 1983b, ApJ, 268, 632.

Keel, W. C. 1983c, ApJ, 269, 466.

Keel, W. C. 1984, ApJ, 282, 75.

Keel, W. C. 1989, AJ, 98, 195.

Kenney, J. D. P., Scoville, N. Z., and Wilson, C. D. 1991, ApJ, 366, 432.

Kennicutt, R. C., Jr. 1988, ApJ, 334, 144.

Kennicutt, R. C., Jr. 1989, ApJ, 344, 685.

Kennicutt, R. C., and Kent, S. M. 1983, AJ, 88, 1094.

Kent, S. M. 1985, ApJS, 59, 115.

Kinney, A. L., Bohlin, R. C., Calzetti, D., Panagia, N., and Wyse, R. F. G. 1993, ApJS, 86, 5 . 
Krabbe, A., Stenberg, A., and Genzel, R. 1994, ApJ, 425, 72.

Krügel, E., Chini, R., Klein, U., Lemke, R., Wielebinski, R., and Zylka, R. 1990, A\&A, 240, 232.

Kuhr, H., Witzel, A., Pauliny-Toth, I. I. K., and Nauber, U. 1981, A\&AS, 45, 367.

Kwan, J., and Xie, S. 1992, ApJ, 398, 105.

Larkin, J. E., Graham, J. R., Matthews, K., Soifer, B. T., Beckwith, S., Herbst, T. M., and Quillen, A. C. 1994, ApJ, 420, 159.

Larkin, J. E., Armus, L., Knop, R. A., Matthews, K., and Soifer, B. T. 1995, ApJ, 452, 599 .

Lauberts, A. 1982, 'The ESO/Uppsala Survey of the ESO(B) Atlas', Munich, European Southern Observatory.

Lester, D. F. 1995, in Proceedings of the Airborne Astronomy Symposium on the Galactic Ecosystem: From Gas to Stars to Dust, ed. M. R. Haas, J. A. Davidson, and E. F. Erickson, A.S.P. Conference Series, Volume 73 (San Francisco: BookCrafters, Inc), 159.

Lester, D. F., Harvey, P. M., and Joy, M. 1986a, ApJ, 302, 280.

Lester, D. F., Harvey, P. M., and Joy, M. 1986b, ApJ, 304, 623.

Lester, D. F., Joy, M., Harvey, P. M., Ellis, H. B., and Parmar, P. S. 1987, ApJ, 321,755 .

Lester, D. F., Zink, E. C., Doppmann, G. W., Gaffney, N. I., Harvey, P. M., Smith, B. J., and Malkan, M. 1995, ApJ, 439, 185.

Lord, S. D., and Young, J. S. 1990, ApJ, 356, 135.

Lord, S. D. 1987, Ph.D. Dissertation, University of Massachusetts.

Nakagawa, T., Nagata, T., Geballe, T. R., Okuda, H., Shibai, H., and Matsuhara, H. 1989, ApJ, 340, 729.

Neff, S. G., and Hutchings, J. B. 1992, AJ, 103, 1746. 
Makinen, P., Harvey, P. M., Wilking, B. A., and Evans, N. J. 1985, ApJ, 299, 341.

Maloney, P., and Black, J. H. 1988, ApJ, 325, 389.

Maloney, P. 1990, The Interstellar Medium in Galaxies, ed. H. Thronson and M. Shull (Boston: Kluwer), 493.

Marsh, K. A., and Helou, G. 1995, ApJ, 445, 599.

Marston, A. P. 1989, AJ, 98, 1572.

Mauersberger, R., Henkel, C., Wielebinski, R., Wiklin, T., and Reuter, H.-P. 1996a, A\&A, in press.

Mauersberger, R., Henkel, C., Whiteoak, J. B., Chin, Y.-N., and Tieftrunk, A. R. 1996b, A\&A, in press.

Meaburn, J., Terrett, D. L., Teokas, A., and Walsh, J. R. 1981, MNRAS, 195, 39.

Minkowski, R., and Osterbrock, D. 1959, ApJ, 129, 583.

Mirabel, I. F., Booth, R. S., Garay, G., Johansson, L. E. B., and Sanders, D. B. 1990, A\&A, 236, 327.

Moorwood, A. F. M., and Glass, I. S. 1984, A\&A, 135, 281.

Moorwood, A. F. M., and Oliva, E. 1988, A\&A, 203, 279.

Moorwood, A. F. M., and Oliva, E. 1994a, ApJ, 429, 602.

Moorwood, A. F. M., and Oliva, E. 1994b, Infrared Phys. Tech., 35, 349.

Morganti, R., Robinson, A., Fosbury, R. A. E., di Serego Alighieri, S., Tadhunter, C. N., and Malin, D. F. 1991, MNRAS, 249, 91.

Morgan, W. W. 1958, PASP, 70, 364.

Nilson, P. 1973, Uppsala General Catalogue of Galaxies, Uppsala, Sweden: Royal Society of Sciences of Uppsala).

Norris, R. P. 1985, MNRAS, 216, 701.

Oliva, E., and Moorwood, A. F. M. 1990, ApJ, 348, L5.

Oliva, E., Salvati, M., Moorwood, A. F. M., and Marconi, A. 1994, A\&A, 288, 457. 
Osmer, P. S., Smith, M. G., and Weedman, D. W. 1974, ApJ, 192, 279.

Osterbrock, D. E. 1989, Astrophysics of Gaseous Nebulae and Active Galactic Nuclei (Mill Valley, CA: University Science Books.

Patnaik, A. R. et al. 1992, MNRAS, 254, 655.

Peimbert, M., and Spinrad, H. 1970, ApJ, 160, 429.

Peimbert, M. and Torres-Peimbert, S. 1981, AJ, 245, 845.

Pence, W. D. 1980, ApJ, 239, 54.

Persson, C. J. L., and Helou, G. 1987, ApJ, 314, 513.

Phillips, A. C. 1993, AJ, 105, 486.

Pogge, R. W. 1989, ApJS, 71, 433.

Prestwich, A. H., Joseph, R. D., and Wright, G. S. 1994, ApJ, 422, 73.

Pritchet, C. 1977, ApJS, 35, 397.

Puxley, P. J., Hawarden, T. G., and Mountain, C. M. 1988, MNRAS, 234, 29P.

Rand, R. J., Kulkarni, S. R., and Rice, W. 1992, ApJ, 390, 66.

Reynolds, J. E. et al. 1994, AJ, 108, 725.

Rice, W., Boulanger, F., Viallefond, F., Soifer, B. T., and Freedman, W. L. 1990, ApJ, 358, 418.

Rice, W., Lonsdale, C. J., Soifer, B. T., Neugebauer, G., Koplan, E. L., Lloyd, L. A., de Jong, T., and Habing, H. J. 1988, ApJS, 68, 91.

Rice, W. 1993, AJ, 105, 67.

Rickard, L. J., and Harvey, P. M. 1984, AJ, 89, 1520.

Rieke, G. H., Cutri, R. M., Black, J. H., Kailey, W. F., McAlary, C. W., Lebofsky, M, J., and Elston, R. 1985, ApJ, 290, 116.

Rieke, G. H., Lebofsky, M. J., Thompson, R. I., Low, F. J., and Tokunaga, A. 1980, ApJ, 238, 24.

Rieke, G. H., Lebofsky, M. J., and Walker, C. E. 1988, ApJ, 325, 679.

Rose, J. A., and Searle, L. 1982, ApJ, 253, 556. 
Rotaciuc, V., Krabbe, A., Cameron, M., Drapatz, S., Genzel, R., Sternberg, A., and Storey, J. W. V. 1991, ApJ, 370, L23.

Rotaciuc, V., Krabbe, A., Cameron, M., Drapatz, S., Genzel, R., Sternberg, A., and Storey, J. W. V. 1991, ApJ, 370, L23.

Rowan-Robinson, M. 1986, MNRAS, 219, 737.

Roy, J.-R., and Belley, J. 1993, ApJ, 406, 60.

Rupen, M. P. 1991, AJ, 102, 48.

Sage, L. J., and Isbell, D. W. A\&A, 247, 320.

Sage, L. J., and Westpfahl, D. J. A\&A, 242, 371.

Saikia, D. J., Unger, S. W., Pendlar, A., Yates, G. J., Axon, D. J., Wolstencroft, R. D., Taylor, K., and Gyldenkerne, K. 1990, MNRAS, 245, 397.

Saikia, D. J., Pedlar, A., Unger, S. W., and Axon, D. J. 1994, MNRAS, 270, 46.

Sandage, A., and Brucato, 1979, AJ, 84, 472.

Sandage, A., and Tammann, G. A. 1981, A Revised Shapley-Ames Catalog of Bright Galaxies (Washington DC: Carnegie Institution of Washington).

Sanders, D. B., and Mirabel, I. F. 1985, ApJ, 298, L31.

Sargent, A. I., Sanders, D. B., and Phillips, T. G. 1989, ApJ, 346, L9.

Schwering, P. B. W., and Israel, F. P. 1989, A\&AS, 79, 79.

Schwering, P. B. W. 1989, A\&AS, 79, 105.

Scoville, N. Z., Sargent, A. I., Sanders, D. B., and Soifer, B. T. 1991, ApJ, 366, L5.

Scoville, N. Z., Soifer, B. T., Neugebauer, G., Young, J. S., Matthews, K., and Yerka, J. 1985, ApJ, 289, 129.

Scoville, N. Z., Thakkar, D., Carlstrom, J. E., and Sargent, A. I. 1993, ApJ, 404, L59.

Scoville, N. Z., and Young, J. S. 1983, ApJ, 265, 148.

Seyfert, C. K. 1943, ApJ, 97, 28. 
Shield, J. C. 1992, ApJ, 399, L27.

Simien, F., and de Vaucouleurs, G. 1986, ApJ, 302, 564.

Sérsic, J. L., and Pastoriza, M. 1965, PASP, 77, 287.

Smith, B. J., Lester, D. F., Harvey, P. M., and Pogge, R. W. 1991, ApJ, 373, 66.

Smith, B. J., Harvey, P. M., Colomé, C., Zhang, C. Y., Di Francesco, J., and Pogge, R. W. 1994, ApJ, 425, 91.

Smith, B. J., Harvey, P. M., and Lester, D. F. 1995, ApJ, 442, 610.

Smith, J. 1982, ApJ, 261, 463.

Smith, J., Harper, D. A., and Loewenstein, R. F. 1984, in Proc. Airborne Astronomy Symposium, ed. H. A. Thronson, Jr. and E. F. Erickson (NASA CP-2353), 277.

Stier, M. T., Traub, W. A., Fazio, G. G., Wright, E. L., and Low, F. J. 1978, ApJ, 226, 347.

Storchi-Bergmann, T., Kinney, A. L., and Challis, P. 1995, ApJS, 98, 103.

Surace, J. A., Mazzarella, J., Soifer, B. T., and Wehrle, A. E. 1993, AJ, 105, 864.

Tacconi, L. J. and Young, J. S. 1989, ApJS, 71, 455.

Talbot, R. J., Jr., Jensen, E. B., and Dufour, R. J. 1979, ApJ, 229, 91.

Telesco, C. M., and Gatley, I. 1981, ApJ, 247, L11.

Telesco, C. M., and Harper, D. A. 1980, ApJ, 235, 392.

Telesco, C. M., Becklin, E. E., Wynn-Williams, C. G., and Harper, D. A. 1984, ApJ, 282, 427.

Telesco, C. M., Dressel, L. L., and Wolstencroft, R. D. 1993, ApJ, 414, 120.

Telesco, C. M., Gatley, I., and Stewart, J. M. 1982, ApJ, 263, L13.

Telesco, C. M., Harper, D. A., and Loewenstein, R. F. 1976, ApJ, 203, L53.

Thompson, R. I., Lebofsky, M. J., and Rieke, G. H. 1978, ApJ, 222, L49.

Turner, J. L., and Ho, P. T. P. 1994, ApJ, 421, 122.

Turner, J. L., Ho, P. T. P., and Beck, S. C. 1987, ApJ, 313, 644. 
Walterbos, R. A. M., and Schwering, P. B. W. 1987, A\&A, 180, 27.

Wainscoat, R. J., de Jong, T., and Wesselius, P. R. 1987, A\&A, 181, 225.

Walker, C. E., Lebofsky, M. J., and Rieke, G. H. 1988, ApJ, 325, 687.

Wall, W. F., Jaffe, D. T., Israel, F. P., and Bash, F. N. 1991, ApJ, 380, 384.

Waller, W. H., Kleinmann, S. G., and Ricker, G. R. 1988, AJ, 95, 1057.

Wamsteker, W., Prieto, A., Vitores, A., Schuster, H. E., Danks, A. C., Gonzales, R., and Rodrigues, 1985, A\&AS, 62, 255.

Ward, M., Penston, M. V., Blades, J. C., and Turtle, A. J. 1980, MNRAS, 193, 563.

Weliachew, L., Casoli, F., and Combes, F. 1988, A\&A, 199, 29.

Whitcomb, S. E., Gatley, I., Hildebrand, R. H., Keene, J., Sellgren, K., and Werner, M. W. 1981, ApJ, 248, 416.

Willner, S. P., Soifer, B. T., Russell, R. W., Joyce, R. R., and Gillett, F. C. 1977, ApJ, 217, L121.

Witt, A. N., Thronson, H. A., and Capuano, J. M., Jr. 1992, ApJ, 393, 611.

Wynn-Williams, C. G., Becklin, E. E., Matthews, K., and Neugebauer, G. 1977, MNRAS, 189, 163.

Wynn-Williams, C. G., Becklin, E. E., Mathews, K., and Neugebauer, G. 1979, MNRAS, 189, 161.

van Driel, W. et al. 1995, AJ, 109, 942.

van Driel, W., de Graaum, Th., de Jong, T., and Wesselius, P. R. 1993, A\&AS, 101, 207.

Van Dyk, S. D., Hyman, S. D., Sramek, R. A., and Weiler, K. W. 1994, IAU Circular 6045, 1.

Véron-Cetty, M.-P., and Véron, P. 1985, A\&A, 145, 425.

Véron-Cetty, M.-P., and Véron, P. 1986, A\&AS, 66, 335. 
Vigotti, M., Grueff, G., Perley, R., Clark, B. G., and Bridle, A. H. 1989, AJ, 98, 419.

Young, J. S. et al. 1995, ApJS, 98, 219.

Young, J. S., Kleinmann, S. G., and Allen, L. E. 1988, ApJ, 334, L63.

Young, J. S., Schloerb, F. P., Kenney, J., and Lord, S. D. 1986, ApJ, 304, 443.

Young, J. S., and Scoville, N. 1982, ApJ, 260, L41.

Young, J. S., Tacconi, L. J., and Scoville, N. Z. 1983, ApJ, 269, 136.

Young, J. S., Xie, S., Kenney, J. D. P., and Rice, W. L. 1989, ApJS, 70, 699. 


\section{CAPTIONS}

Figure 1. The co-added scan data. For the NGC 3256 and Uranus 5/23/90 data, which were acquired with the 20 channel system, only the data from the central 8 channels in the first bank of detectors are displayed. The orientation of the array on the sky and the scan direction are indicated by the coordinates on the plots and by the rotation angle in Table 2. The detectors are separated by $13^{\prime \prime} .8$ at $100 \mu \mathrm{m}$ and $7^{\prime \prime} .0$ at $50 \mu \mathrm{m}$.

Figure 2. The galaxy scan data for the peak channel, plotted with the data for the point source object from the same flight. The curves with the larger error bars are the galaxy profiles.

Figure 3. Far-infrared profiles along the long axis of the array, obtained from averaging the central $22^{\prime \prime}(100 \mu \mathrm{m})$ or $10^{\prime \prime}(50 \mu \mathrm{m})$ of the summed scans for each detector. The data for the point source object from the same flight (open squares) is displayed with the galaxy data (filled circles).

Figure 4. The nod data. The top panel displays the data from the first bank of detectors, while the lower panel shows the data from the second bank. Both the galaxy data and the corresponding point source profile are shown. In Figure 4d, we display the same M 83 and $\eta$ Carinae data that are shown in Figure 4c, but with the end channels set to zero. During the NGC 891 observations, the array was shifted along its minor axis, giving two overlapping sets of data. For NGC 7331, three overlapping sets of data were obtained.

Figure 5. The major axis $100 \mu \mathrm{m}$ profile for NGC 7331, along with the $23^{\prime \prime}$ resolution CO $(1-0)$ observations of Braine et al. (1993; 1995, private communication). The $(0,0)$ point of the Braine et al. CO data is offset from the optical and radio continuum of NGC 7331 (Table 1); from their partial map, we have selected the positions closest to our observed locations for comparison (within $5^{\prime \prime}$ ). This plot also contains 3 profiles derived from the Pogge (1989) 
$\mathrm{H} \alpha+[\mathrm{N} \mathrm{II}]$ image: 1) a cut across the unsmoothed array along our array position, 2) a cut along our array position after the image has been smoothed to our beamsize, and 3) a cut along our array location after the central bulge and nuclear emission has been removed and the image smoothed.

Figure 6. $\log \mathrm{I}(\mathrm{CO})$ vs. $\log \tau_{100}$. The data for the galaxies with strong star formation and/or Seyfert activity within the KAO beam are plotted as asterisks, while the more quiescent galaxies are plotted as filled triangles. The plotted curves show expected relationships using the standard $\mathrm{I}(\mathrm{CO}) / \mathrm{n}\left(\mathrm{H}_{2}\right)$ ratio (Bloemen et al. (1986) and the Makinen et al. (1985) $\mathrm{A}_{\mathrm{V}} / \tau_{100}$ relationship (see text). Note that, for the galaxies without small beam $50 \mu \mathrm{m}$ measurements and $\mathrm{T}_{d}$ (bulge) $>\mathrm{T}_{d}$ (global), the plotted values of $\tau_{100}$ are upper limits (see Table 6 ).

Figure 7. $\log \mathrm{I}(\mathrm{CO})$ vs. the $\log \mathrm{A}_{\mathrm{V}}$ derived from the Balmer decrement. Symbols are as in Figure 6.

Figure 8. $\log \mathrm{I}(\mathrm{CO})$ vs. the $\log \mathrm{A}_{\mathrm{V}}$ derived from $\mathrm{F}(\mathrm{H} \alpha) / \mathrm{F}(\mathrm{Br} \gamma)$. Symbols are as in Figure 6.

Figure 9. $\log \mathrm{L}(\mathrm{B}) / \Omega$ vs. $\log \mathrm{L}(\mathrm{FIR}) / \Omega$. Symbols are as in Figure 6 .

Figure 10. $\log \mathrm{L}(\mathrm{FIR}) / \Omega$ vs. $\log \mathrm{I}(\mathrm{CO})$. Symbols are as in Figure 6 .

Figure 11. $\log \mathrm{L}(\mathrm{FIR}) / \mathrm{L}(\mathrm{B})$ vs. $\log \mathrm{I}(\mathrm{CO})$. Symbols are as in Figure 6.

Figure 12. $\log \mathrm{L}(\mathrm{FIR}) / \mathrm{L}(\mathrm{H} \alpha)$ vs. $\log \mathrm{I}(\mathrm{CO})$. Symbols are as in Figure 6. The plotted curve is the line expected for dust heating by OB stars, assuming a Salpeter IMF and the standard Galactic $\mathrm{I}(\mathrm{CO}) / \mathrm{n}\left(\mathrm{H}_{2}\right)$ ratio (Bloemen et al. 1986).

Figure 13. $\log \mathrm{L}(\mathrm{FIR}) / \mathrm{L}(\mathrm{H} \alpha)$ vs. $\tau_{100}$. Symbols are as in Figure 6. The plotted curve is the line expected for dust heating by OB stars, assuming a Salpeter IMF and $\mathrm{A}_{\mathrm{V}} / \tau_{100}$ (Makinen et al. 1985).

Figure 14. $\log \mathrm{L}(\mathrm{FIR}) / \mathrm{L}(\mathrm{Br} \gamma)$ vs. $\mathrm{I}(\mathrm{CO})$. Symbols are as in Figure 6. The plotted curve is the line expected for dust heating by OB stars, as in Figure 12. 
Figure 15. $\log \mathrm{L}(\mathrm{FIR}) / \mathrm{L}(\mathrm{Br} \gamma)$ vs. $\tau_{100}$. Symbols are as in Figure 6. The plotted curve is the line expected for dust heating by OB stars, as in Figure 13.

Figure 16. Log $\mathrm{L}(\mathrm{FIR}) / \mathrm{L}(\mathrm{H})$ vs. $\mathrm{I}(\mathrm{CO})$. Symbols are as in Figure 6. The solid curve is the relationship expected by extinction using the assumptions given in the text. It is normalized to $\mathrm{L}(\mathrm{FIR}) / \mathrm{L}(\mathrm{H}) \sim 2$ at $\mathrm{A}_{\mathrm{V}}=0$, which corresponds to a situation where $\sim 1 / 5$ th of the total bolometric luminosity of the stars is absorbed by dust and re-emitted in the far-infrared, assuming a K5 giant stellar population.

Figure 17. $\log \mathrm{L}(\mathrm{H} \alpha) / \mathrm{L}(\mathrm{H})$ vs. $\mathrm{I}(\mathrm{CO})$. Symbols are as in Figure 6 . 